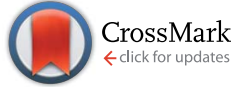

Cite this: RSC Adv., 2015, 5, 100809

Received 8th October 2015

Accepted 17th November 2015

DOI: $10.1039 / \mathrm{c} 5 \mathrm{ra} 20878 \mathrm{e}$

www.rsc.org/advances

\section{Evidence of a correlation between magnetic and structural transitions in $\mathrm{Y}_{2-x} \mathrm{Zn}_{x} \mathrm{Ru}_{2} \mathrm{O}_{7}$ pyrochlore compounds $\dagger$}

\author{
Giulia Berti, ${ }^{a}$ Samuele Sanna, ${ }^{b}$ Rocío Ruiz-Bustos, ${ }^{c}$ Joost van Duijn, ${ }^{d}{ }^{d}$ \\ Alberto Brambilla, ${ }^{a}$ Álvaro Muñoz-Noval, $\S^{e}$ Francesco Demartin, ${ }^{f}$ Lamberto Duò ${ }^{a}$ \\ and Carlo Castellano*f
}

\begin{abstract}
We report on an experimental investigation of the magnetic and structural properties of the $\mathrm{Y}_{2-x} \mathrm{Zn}_{x} \mathrm{Ru}_{2} \mathrm{O}_{7}$ pyrochlore system as a function of the $Z n$ doping in the range $0.00 \leq x \leq 0.20$. Magnetization and muon spin relaxation measurements show that the antiferromagnetic transition typical of the undoped compound at $T_{\mathrm{N}}=77 \mathrm{~K}$ is slightly reduced and broadened in the $\mathrm{Zn}$ doped sample. Extended $\mathrm{X}$-ray absorption fine structure measurements show a peak in the $\mathrm{Ru}-\mathrm{O}(1)$ first shell Debye-Waller factor at a temperature $T_{\mathrm{DW}} \approx T_{\mathrm{N}}$, giving evidence for a strong magnetoelastic coupling detected up to $x=0.10$. A local precursor order-disorder transition at $T^{*}>T_{N}$ is also observed up to $x=0.10$. For $x>0.10$ these local structural features are hidden due to the increasing local lattice distortion induced by the $\mathrm{Zn}^{2+}$ dopant whereas the magnetic transition is still present.
\end{abstract}

\section{Introduction}

The pyrochlore structure corresponds to the general chemical formula $\mathrm{A}_{2} \mathrm{~B}_{2} \mathrm{O}(1)_{6} \mathrm{O}(2)$, where $\mathrm{A}$ is a trivalent (or divalent) rare earth or $\mathrm{Y}$ ion and $\mathrm{B}$ is a tetravalent (or pentavalent) transition metal ion. The structure is cubic crystallizing in the space group $\mathrm{Fd} 3 \mathrm{~m}$ and all the atoms are in special positions. The oxygens occupy the Wyckoff sites $48 \mathrm{f}[\mathrm{O}(1)]$ and $8 \mathrm{~b}[\mathrm{O}(2)]$ at the vertices of the $\mathrm{BO}_{6}$ octahedra and bridging the $\mathrm{Y}$ atoms to form chains, respectively; $1 / 8$ of the anions are absent giving rise to oxygen vacancies at the $8 \mathrm{a}$ sites. The only variable coordinate is $\bar{x}$, describing the position of the $O(1)$ atoms $(\bar{x}, 1 / 8,1 / 8)$ and determining the distortion degree of the $\mathrm{BO}(1)_{6}$ corner sharing

${ }^{a}$ Dipartimento di Fisica, Politecnico di Milano, piazza Leonardo da Vinci 32, 20133 Milano, Italy

${ }^{b}$ Dipartimento di Fisica and CNISM, Università di Pavia, viale Bassi 6, 27100 Pavia, Italy

${ }^{c}$ Departamento de Mecánica, Universidad de Córdoba, 14071 Córdoba, Spain

${ }^{d}$ Instituto de Investigación en Energías Renovables, Departamento de Física Aplicada, Universidad de Castilla-La Mancha, 02006 Albacete, Spain

ICMM-CSIC and Spanish CRG, European Synchrotron Radiation Facility (ESRF), 71 Avenue des Martyrs, 38000 Grenoble, France

${ }^{f}$ Dipartimento di Chimica, Università degli Studi di Milano, via Golgi 19, 20133 Milano, Italy. E-mail: carlo.castellano@unimi.it

$\dagger$ Electronic supplementary information (ESI) available: Details on $\mu$ SR analysis. See DOI: 10.1039/c5ra20878e

\$ Present address: Universidad de Córdoba, Campus Universitario de Rabanales, Edificio Leonardo da Vinci, 14071 Córdoba, Spain.

$\S$ Present address: Department of Applied Chemistry, Hiroshima University, 1-4-1 Kagamiyama, Higahi-Hiroshima, Hiroshima 739-8527, Japan. octahedra with respect to the ideal undistorted pyrochlore structure. ${ }^{1}$ The wide variety of compositions and dopings of these strongly correlated electron systems makes them good candidates for several potential applications as insulators, ion and electron conductors, superconductors, catalysts, robust nuclear waste forms, ion exchangers, phosphors and solid-oxide fuel cells (SOFC) cathodes. ${ }^{2,3}$ Within this wide family of complex oxides, ruthenium-containing pyrochlores $\mathrm{A}_{2} \mathrm{Ru}_{2} \mathrm{O}_{7}$ have recently received renewed attention due to the multitude of their interesting structural, electrical and magnetic properties mainly related to short-range ordered phases peculiar of geometrically frustrated magnets such as spin glasses and spin ices. ${ }^{1,4-6} \quad \mathrm{Y}_{2} \mathrm{Ru}_{2} \mathrm{O}_{7}$ exhibits a low spin state $(S=1)$ with a remarkable antiferromagnetic (AFM) coupling of $\mathrm{Ru}^{4+}$ with a Néel temperature $\left(T_{\mathrm{N}}=77 \mathrm{~K}\right)$ higher than in other pyrochlore compounds. The combination of geometrical frustration, spinglass state and AFM correlation for this composition should result in symmetry lowering already at $T \simeq T_{\mathrm{N}} \cdot{ }^{1}$ The cited properties include also metal-insulator as well as spin-singlet transitions. ${ }^{7-11}$ A very good electrochemical behavior of $\mathrm{Ru}-$ based pyrochlores has been observed at temperatures as low as $350{ }^{\circ} \mathrm{C}$, which has motivated their study as intermediate temperature SOFC (IT-SOFC) cathodes. ${ }^{12}$ The greater part of the literature published to date concerns materials in which $\mathrm{Ru}$ is either $4+\left(\right.$ with $\mathrm{A}^{3+}$ ) or $5+\left(\right.$ with $\mathrm{A}^{2+}$ ) while few studies are reported on the effect of a mixed oxidation state at the A site. Chemical doping at the A site with divalent cations would also imply a hole doping effect, expectedly providing these systems with the electronic conductivity required to overcome the Mott-Hubbard threshold. ${ }^{13}$ This effect, coupled with ionic 
conductivity due to vacancy disorder, makes doped $\mathrm{Y}_{2} \mathrm{Ru}_{2} \mathrm{O}_{7}$ even more prospective cathode materials for IT-SOFC. ${ }^{\mathbf{1 4}}$ Moreover in pyrochlores, cation or anion disorder may give rise to order-disorder transitions influencing also the conductivity and the presence of metal-insulator transitions., ${ }^{75-17}$ This phenomenon is coherent with the pyrochlores predisposition towards full or partial disorder, structural distortion and phase transition from pyrochlore to a defect fluorite structure. ${ }^{6,18}$ In this general framework, doping $\mathrm{Y}_{2} \mathrm{Ru}_{2} \mathrm{O}_{7}$ with $\mathrm{Zn}^{2+}$ results in mixed charge at both $\mathrm{A}$ and $\mathrm{B}$ cationic sites and consequently a ruthenium mixed valence $4+/ 5+$ is present. In $\mathrm{Zn}$-doped samples $\mathrm{Y}_{2-x} \mathrm{Zn}_{x} \mathrm{Ru}_{2} \mathrm{O}_{7}$ (with $x=0.10,0.15$ and 0.20 ) a relevant role from the structural point of view is played by the smaller ionic radius of the $\mathrm{Zn}^{2+}(0.90 \AA)$ ions with respect to that of $\mathrm{Y}^{3+}(1.02 \AA)$. As already observed in related A site doped systems like $\mathrm{Bi}_{2-x} \mathrm{Y}_{x} \mathrm{Ru}_{2} \mathrm{O}_{7}$, by increasing the smaller ion doping level $x$, the $\mathrm{Ru}-\mathrm{O}(1)-\mathrm{Ru}$ bond angle decreases, the $\mathrm{Ru}-\mathrm{O}(1)$ interatomic distance increases, the bend in the $\mathrm{RuO}(1)_{6}$ corner sharing zig-zag chains and consequently the octahedron distortion increase. ${ }^{19}$ This doping effect influences also the conductivity which results to be metallic when $x$ is lower than 1.2 and consequently the $\mathrm{Ru}-\mathrm{O}(1)-\mathrm{Ru}$ bond angle is greater than $133^{\circ} \cdot .^{19,20}$ The same stereochemical result is observed also in $\mathrm{Bi}_{2-x} \mathrm{Nd}_{x} \mathrm{Ru}_{2} \mathrm{O}_{7-\delta}, \mathrm{Bi}_{2-x} \mathrm{Yb}_{x} \mathrm{Ru}_{2} \mathrm{O}_{7-\delta}, \mathrm{Pb}_{2-x} \mathrm{Y}_{x} \mathrm{Ru}_{2} \mathrm{O}_{7-\delta}$ and for other chemical compositions. ${ }^{\mathbf{4 , 2 0 2}}$ The cited change in the conduction behavior from semiconductor to metal is confirmed also by thermoelectric and Seebeck coefficient measurements as a function of doping and temperature indicating that the major carriers are holes. ${ }^{22}$ It is worth noting that in $\mathrm{Zn}^{2+}$ doped $\mathrm{Bi}_{1.50} \mathrm{Zn}_{0.50} \mathrm{Ru}_{2} \mathrm{O}_{6.75}$ compounds an anomalous, unique and not yet clarified electrical and magnetic behavior among the known electronic ground states of $\mathrm{Ru}^{4+}$ pyrochlores was observed. ${ }^{5,9} \mathrm{On}$ the other hand, in the case of $\mathrm{Y}_{2-x} \mathrm{Ca}_{x} \mathrm{Ru}_{2} \mathrm{O}_{7}$ it has been shown that the substitution of $\mathrm{Y}^{3+}$ by the larger $\mathrm{Ca}^{2+}$ significantly influences both the magnetic and the conductive properties. ${ }^{15}$ At present, there is not an exhaustive view of the effective role of the A site doping since this depends on the chemical nature, valence and occupancy of dopants, and on their ionic radius, magnetic configuration and stereochemical impact. In a recent EXAFS experiment on the related undoped compound $\mathrm{Y}_{2} \mathrm{Ru}_{2} \mathrm{O}_{7},{ }^{6}$ we found evidence of: (i) a large magnetoelastic coupling pointed out by a huge and broad peak in the DebyeWaller factor $\sigma^{2}$ for the $\mathrm{Ru}-\mathrm{O}(1)$ first coordination shell in the temperature region around the antiferromagnetic transition at $T_{\mathrm{N}} \simeq 77 \mathrm{~K}$; (ii) an axial distortion of the $\operatorname{RuO}(1)_{6}$ octahedra above a critical temperature $T^{*} \simeq 150 \mathrm{~K}$ which was attributed to a precursor order-disorder (OD) transition. Local octahedron distortion also appeared in the $\mathrm{Ru}-\mathrm{Ru}$ and $\mathrm{Ru}-\mathrm{Y}$ distances which, respectively, increase and decrease at $T>T^{*}$. Still, neutron diffraction measurements at $T=10,100$, and $295 \mathrm{~K}$ indicated that the $F d 3 \mathrm{~m}$ symmetry is retained also below $T_{\mathrm{N}}{ }^{23,24}$ These results pointed out the presence of a local structural distortion and additional disorder developing as a function of temperature, probably further influenced in $\mathrm{Y}_{2-x} \mathrm{Zn}_{x} \mathrm{Ru}_{2} \mathrm{O}_{7}$ by the doping level at the rare earth site. In this context, the aim of the present work is to characterize the effect of the cation disorder and of the $\mathrm{Ru}$ mixed valence induced by the A site doping on the local structure of the $\mathrm{Ru}-\mathrm{Ru}$ and $\mathrm{Ru}-\mathrm{Y}$ second coordination shell and of the $\mathrm{RuO}(1)_{6}$ first shell octahedra.

\section{Experimental}

A powdered sample of $\mathrm{Y}_{2-x} \mathrm{Zn}_{x} \mathrm{Ru}_{2} \mathrm{O}_{7}$ was prepared using the solid state reaction method. Mixtures of $\mathrm{Y}_{2} \mathrm{O}_{3}, \mathrm{ZnO}$ and $\mathrm{RuO}_{2}$ in proper molar ratio were intimately mixed, pressed into pellets and reacted at $1000-1200{ }^{\circ} \mathrm{C}$ in air with intermediate grindings. The sample was characterized by powder X-ray diffraction using a Philips XPert Pro diffractometer with a copper source and incident beam monochromator, revealing it to be single phase. Rietveld analysis confirmed the cubic pyrochlore structure. DC magnetization measurements were performed by means of a superconducting quantum interference device (SQUID) magnetometer (Quantum Design) on all the tested samples. Muon-spin relaxation measurements were carried out at the GPS spectrometer ( $\pi \mathrm{M} 3$ beam line) at the Paul Scherrer Institut (PSI), Villigen, Switzerland. The extended X-ray absorption fine structure (EXAFS) measurements were carried out at the $\mathrm{Ru}$ K-edge (22 $117 \mathrm{eV}$ ) in transmission geometry at the European Synchrotron Radiation Facility (ESRF) in Grenoble (France) on beamline BM25A, using a double $\mathrm{Si}(111)$ crystal monochromator of pseudo channel-cut type refrigerated at $200 \mathrm{~K}$ by a homemade ethanol cooling system. The energy resolution was close to $1.2 \mathrm{eV}$. The measurements were performed at 24 different temperatures between 8 and $298 \mathrm{~K}$. An Oxford CV-F optistat liquid helium cryostat with a LHe Dewar supply was used. The EXAFS data were reduced applying standard procedures using the Demeter package ${ }^{25}$ and fits on $k^{3}$ weighted signals were carried out in $r$ space using theoretical functions from FEFF9 code. $^{26}$

\section{Results and discussion}

Fig. 1 shows the lattice parameter as a function of the nominal $\mathrm{Zn}$ content for the $\mathrm{Y}_{2-x} \mathrm{Zn}_{x} \mathrm{Ru}_{2} \mathrm{O}_{7}$ samples, as determined by $\mathrm{X}$ ray diffraction measurements. The data show that the lattice parameter is sizeably reduced for a low content of $\mathrm{Zn}, x=0.10$. However, the lattice parameter keeps nearly constant by further increasing the $\mathrm{Zn}$ content up to $x=0.50$. A full analysis of the diffraction data performed on the same samples, ${ }^{\mathbf{1 4}}$ shows that the solution limit of the $\mathrm{Y} / \mathrm{Zn}$ substitution is close to the nominal value of $x=0.20$. In order to further study the structural evolution as a function of $\mathrm{Zn}$ content we have employed the EXAFS technique, which is a powerful tool to investigate the structure on a local or short-range scale around the absorber. Typical EXAFS signals in the 8-298 $\mathrm{K}$ temperature range are shown in Fig. 2 up to $15.5 \AA^{-1}$ for different Zn contents. Fig. 3 shows the representative Back Fourier transform best-fit curve and single contributions for spectrum taken at $8 \mathrm{~K}$ for $x=0.10$.

In fitting the data (full $r$ fit range: 1.05-3.80 $⿱$ ) , one $\mathrm{Ru}-\mathrm{O}(1)$ first shell with coordination number $N=6$, the $\mathrm{Ru}-\mathrm{Ru}$ and $\mathrm{Ru}-\mathrm{Y}$ second shell peaks $(N=6$, each), two other longer $\mathrm{Ru}-\mathrm{O}(1)$ peaks ( $N=6$ and 12 , respectively) and two multiscattering peaks were used (Fig. 3). These last two peaks included in the fit were the triple forward scattering through the absorber $\mathrm{Ru}-\mathrm{O}(1)-\mathrm{Ru}-$ 


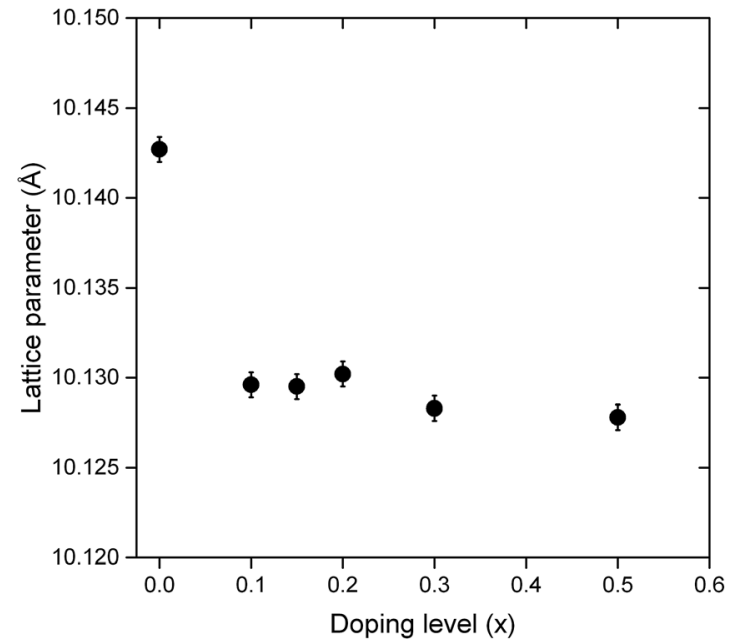

Fig. 1 Lattice parameter as a function of the nominal $\mathrm{Zn}$ content for the $\mathrm{Y}_{2-x} \mathrm{Zn}_{x} \mathrm{Ru}_{2} \mathrm{O}_{7}$ samples (data from ref. 14).

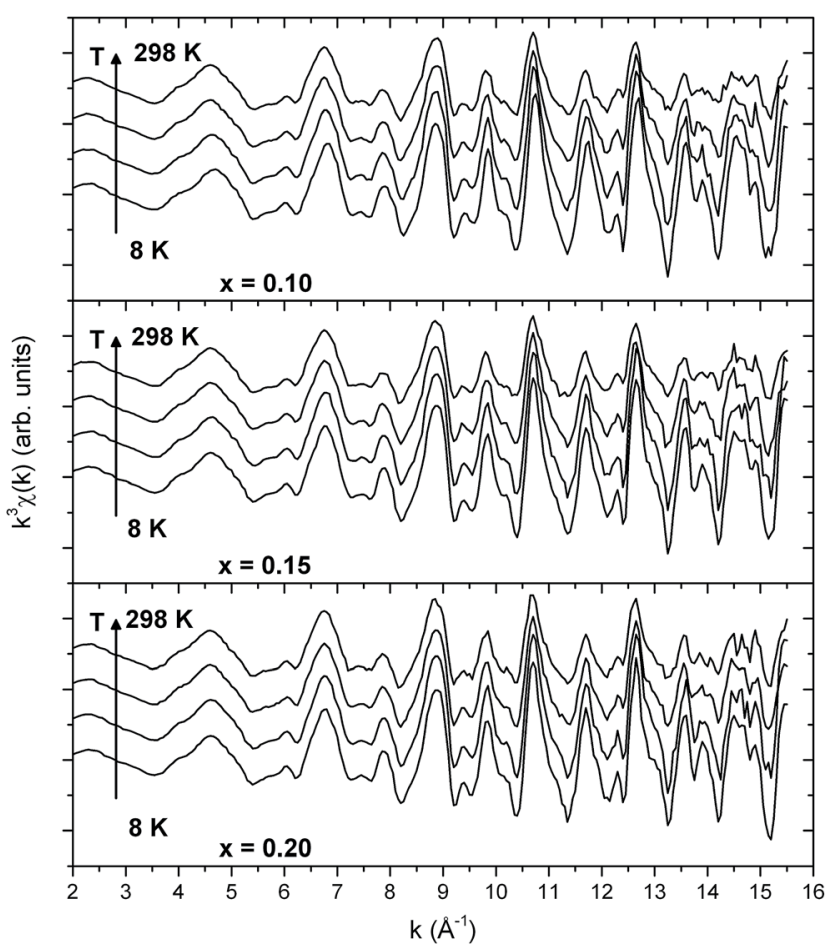

Fig. 2 Examples of typical EXAFS signals in the 8-298 $\mathrm{K}$ temperature range for different doping levels $(x)$. Data were offset for clarity.

$\mathrm{O}(1)(N=6)$ and the double scattering $\mathrm{Ru}-\mathrm{O}(1)-\mathrm{O}(1)(N=24)$. The initial fitting parameters were the mean square relative displacements $\sigma^{2}$ of the absorber and backscattering atoms, $\uparrow$ the interatomic bond lengths $R$, the $E_{0}$ shift in the edge energy with respect to the theoretical value and the amplitude reduction factor $S_{0}{ }^{2}$ from multielectron effects. This last factor is generally between 0.8 and 1.0. At low $T$ and doping, the lattice is well ordered and $S_{0}{ }^{2}$ was determined from the average of several

I In the present work the Debye-Waller factor is intended as the mean square relative displacement.

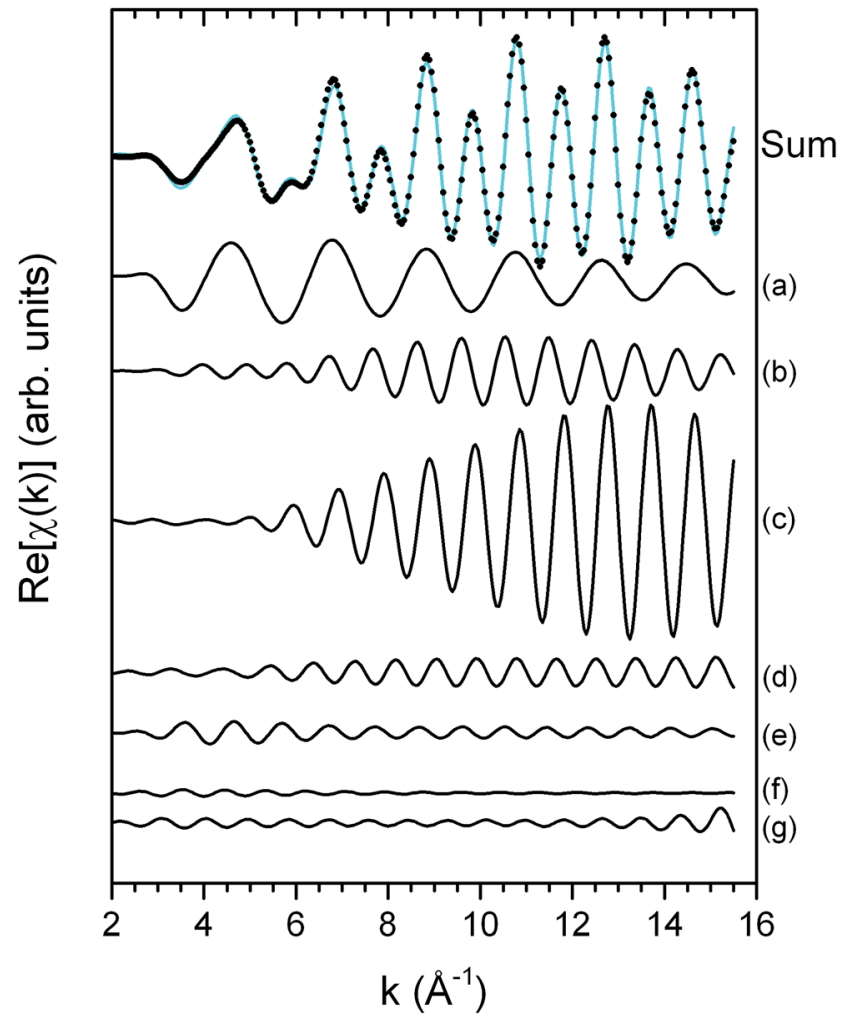

Fig. 3 Back Fourier transform best-fit curve and single contributions for spectrum taken at $8 \mathrm{~K}$ on sample with $x=0.10$. Letters refer to different paths as follows: (a) Ru-O1.1-Ru; (b) Ru-Ru1.1-Ru; (c) $\mathrm{Ru}-\mathrm{Y} 1.1-\mathrm{Ru}$; (d) $\mathrm{Ru}-\mathrm{O} 1.2-\mathrm{Ru}$; (e) $\mathrm{Ru}-\mathrm{O} 1.1-\mathrm{Ru}-\mathrm{O} 1.1-\mathrm{Ru}$; (f) $\mathrm{Ru}-\mathrm{O} 1.3-\mathrm{Ru}$; (g) Ru-O1.1-O1.3-Ru. Data were offset for clarity.

fits to scans at $8 \mathrm{~K}$ and fixed at 0.96 . The $E_{0}$ shift was also constrained to a single value for all the paths and the coordination numbers were fixed to the values reported above for the first shell and to the crystallographic values for the remaining paths.

In the bottom panels of Fig. 4 the Ru-O(1) first shell mean square relative displacement $\sigma^{2}$ is displayed as a function of temperature for nominal $\mathrm{Zn}$ content in the range $x=0.00-0.20$. The data of the undoped $x=0$ compound are from ref. 6 . A clear peak of $\sigma^{2}(T)$ is observed for the $x=0.00$ and 0.10 sample, that occurs at the same temperature below which the zero field cooled (ZFC) and field cooled (FC) susceptibility curves (top panels Fig. 4) get separated, i.e. where a canted AFM order is established in the sample. We have recently pointed out for $x=0$ (ref. 6) that this behaviour gives evidence of a significant magnetoelastic coupling on a local scale, marked by a peak in the low temperature side of the $\sigma^{2}$ graph at a temperature $T_{\mathrm{DW}}$. This phenomenon is still observed for $x=0.10$, even if the $\sigma^{2}(T)$ peak is weaker than in the undoped sample. Fig. 4 shows that this peak completely disappears (i.e., $T_{\mathrm{DW}}$ vanishes) when the Zn doping is further increased for $x \geq 0.15$ even if the ZFC and FC curves still display a separation (indicated by the dashed line in Fig. 4).

In order to clarify the magnetic state of the $\mathrm{Zn}$ doped compounds we have performed zero field muon spin relaxation (ZF- $\mu \mathrm{SR}$ ) measurements for $x=0.20$. The details of the ZF- $\mu \mathrm{SR}$ 


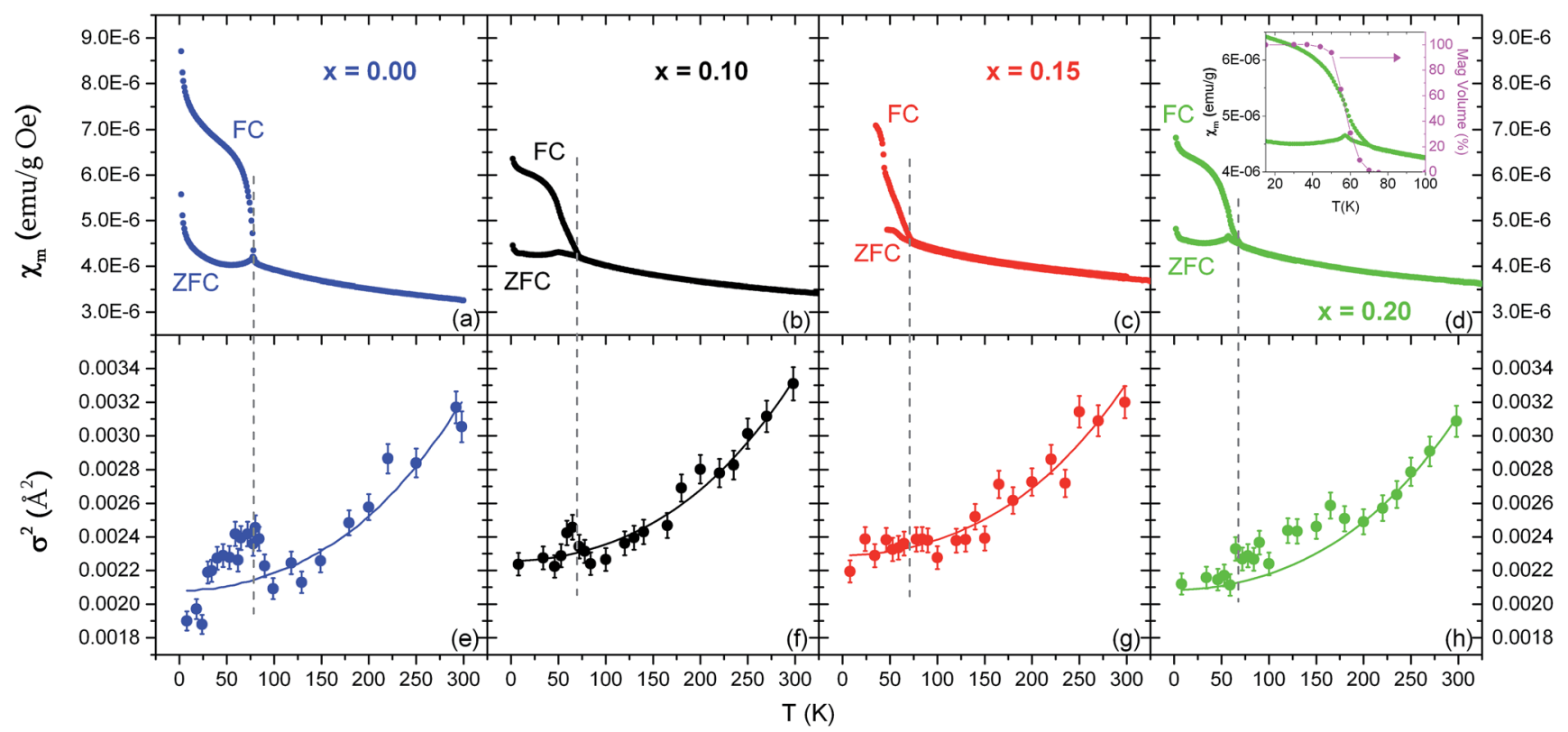

Fig. 4 Upper row: magnetic susceptibility as a function of temperature in both ZFC and FC conditions with an external applied field $\mu_{0} H=0.1 \mathrm{~T}$ for the undoped compound (data previously published) and three different doping levels. Lower row: Ru-O(1) first shell mean square relative displacement $\sigma^{2}\left(\AA^{2}\right)$ as a function of temperature for the undoped compound (data previously published) and three different doping levels (the continuous lines are fits to a correlated Debye model). Vertical dashed lines mark the position of the $T_{\mathrm{N} \text {, onset }}$ in comparison with the peaks in the $\sigma^{2}$ graphs. Inset: magnetic volume as a function of temperature for the sample with $x=0.20$.

analysis are reported in the ESI section. $\dagger$ A clear signature of the emergence of the AFM state is given by the appearance of oscillations of the muon asymmetry at low temperature (see Fig. ESI $\dagger$ ). These oscillations reflect coherent precession of the muon spin around spontaneously ordered magnetic moments. A direct comparison of the temperature dependence of the AFM volume fraction, as detected by ZF- $\mu \mathrm{SR}$, and of the magnetic susceptibility is displayed in the inset of Fig. $4 \mathrm{~d}$. The data show that the magnetic volume starts to increase at the same temperature below which the susceptibility curves get separated, i.e. this temperature can be considered as the onset of the magnetic transition $T_{\mathrm{N} \text {,onset. }}$. In addition, one can notice that the cusp in the ZFC curve appears at the temperature corresponding to nearly $50 \%$ of the magnetic volume fraction, i.e. the temperature of the cusp can be considered as the average of the magnetic transition, $T_{\mathrm{N}, \mathrm{av}}$. Both $T_{\mathrm{N}, \text { onset }}$ and $T_{\mathrm{N}, \mathrm{av}}$ are diplayed in Fig. 5 as a function of the $\mathrm{Zn}$ doping. The results show that $T_{\mathrm{N}, \text { onset }}$ (squares) is systematically higher than $T_{\mathrm{N}, \text { av }}$ (triangles) for the $\mathrm{Zn}$ doped samples. This indicates that the presence of the $\mathrm{Zn}$ doping broadens the magnetic transition with respect to the undoped compound, for which $T_{\mathrm{N} \text {,onset }}=T_{\mathrm{N}, \mathrm{av}}$. Actually, in the undoped compound the temperature dependence of the magnetic volume fraction measured by ZF- $\mu$ SR displays a very sharp magnetic transition ${ }^{27}$ whilst the transition width on the $x$ $=0.20$ sample is of about $15 \mathrm{~K}$ (inset of Fig. $4 \mathrm{~d}$ ). Noteworthy, these data confirm that the magnetic transition is still at play also when the $\sigma^{2}(T)$ peak is absent $(x \geq 0.15)$. The disappearance of the $\sigma^{2}(T)$ peak at the $T_{\mathrm{N} \text {,onset }}$ with increasing doping is likely due to a weakening or hiding of the magnetoelastic coupling and an increase in the local lattice distortion induced by the $\mathrm{Zn}^{2+}$ smaller ions. In agreement with this doping related behaviour, we point out also an anomalous local disorder at temperatures lower than about $200 \mathrm{~K}$ for $x=0.20$ (see Fig. $4 \mathrm{~h}$ ), slightly deviating from a correlated Debye trend.

In general in these systems, the $\sigma^{2}$ parameter follows a temperature dependent correlated Debye-like behavior $\sigma_{\mathrm{D}}{ }^{2}$ at high temperatures, with a correlated Debye temperature $\theta_{\mathrm{D}}$ around $800 \mathrm{~K}$. Moreover, a temperature independent static contribution $\sigma_{\mathrm{S}}{ }^{2}$ due to the presence of a possible distorted environment takes place. The Debye-Waller factor shown in Fig. 4 for the three doping levels was fitted above $120 \mathrm{~K}$

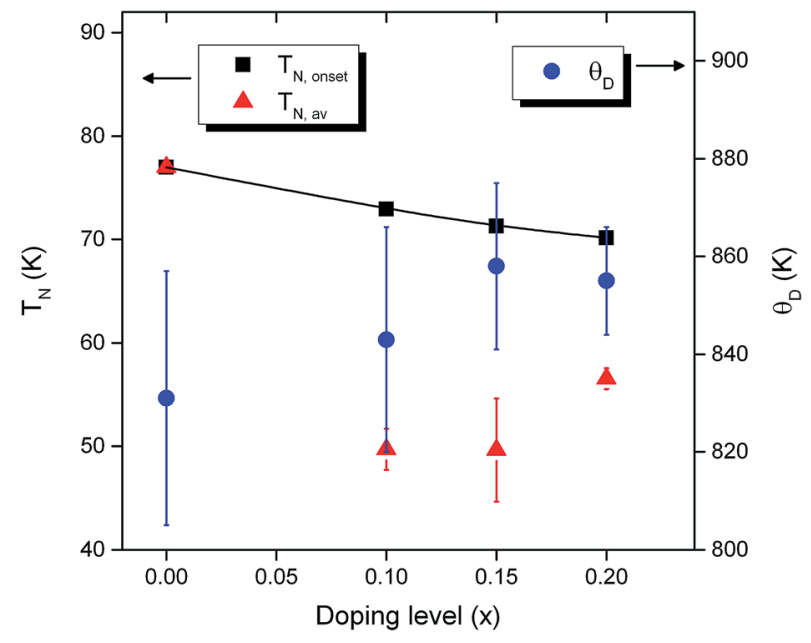

Fig. 5 Onset and average transition temperatures, $T_{\mathrm{N} \text {,onset }}$ (squares) and $T_{\mathrm{N} \text {, av }}$ (triangles), and correlated Debye temperature as a function of the nominal $\mathrm{Zn}$ content for the $\mathrm{Y}_{2-x} \mathrm{Zn}_{x} \mathrm{Ru}_{2} \mathrm{O}_{7}$ samples. 
Table 1 Correlated Debye temperature and static offset $\left(\sigma_{\mathrm{S}}{ }^{2}\right)$ as retrieved from the Debye fit curves shown in Fig. 4. Data for the undoped $x=0.00$ sample are taken from ref. 6

\begin{tabular}{lllll}
\hline & $x=0.00$ & $x=0.10$ & $x=0.15$ & $x=0.20$ \\
\hline$\theta_{\mathrm{D}}(\mathrm{K})$ & $831(26)$ & $843(23)$ & $858(17)$ & $855(11)$ \\
$\sigma_{\mathrm{S}}{ }^{2}\left(\AA^{2}\right)$ & $-0.0002(3)$ & $0.0000(3)$ & $-0.0001(3)$ & $0.0000(3)$
\end{tabular}

considering $\sigma^{2}(T)=\sigma_{\mathrm{D}}{ }^{2}+\sigma_{\mathrm{S}}{ }^{2}$, as already shown in previous papers, ${ }^{28,29}$ where this sum is usually a good approximation for all phonon modes. ${ }^{30,31}$

The fit parameters were the $\theta_{\mathrm{D}}$, which is a measure of the $\mathrm{Ru}-\mathrm{O}$ bond strength, in accordance with other pyrochlores, ${ }^{32}$ and $\sigma_{\mathrm{S}}^{2}$; the obtained data are reported in Table 1, for all the compositions. The values of $\theta_{\mathrm{D}}$ for the samples are the same within the estimated errors while the static contributions are all very small (see Table 1 and Fig. 5).

In the undoped $\mathrm{Y}_{2} \mathrm{Ru}_{2} \mathrm{O}_{7}$ compound a splitting of both the (i) $\mathrm{Ru}-\mathrm{Y}$ and $\mathrm{Ru}-\mathrm{Ru}$ distances and (ii) $\mathrm{Ru}-\mathrm{O}(1) 1^{\text {st }}$ and $2^{\text {nd }}$ subshells has been recently observed ${ }^{6}$ above a temperature $T^{*} \approx$ $2 T_{\mathrm{N}}$ by EXAFS (data reproduced in Fig. 6a). This behaviour indicates the appearance of a local distortion at a characteristic temperature $T^{*}$, where a possible local order-disorder transition occurs. Following the analysis performed on the undoped material, ${ }^{6}$ we have performed the same fitting procedure also for the $\mathrm{Zn}$ doped compounds, by replacing the $\mathrm{Ru}-\mathrm{O}(1)$ first shell path with two first sub-shells Ru-O(1) (with coordination numbers $N_{\text {shorter }}=4$ and $N_{\text {longer }}=2$ ). It is important to underline that when a peak splitting $\Delta R$ occurs, the EXAFS ability to resolve two different peaks is intrinsically limited by the general rule $\Delta R>\pi /\left(2 k_{\max }\right)$, being here $k_{\max }=15.5 \AA^{-1}$. $^{33,34}$ For this reason some caution must be taken when results beyond this theoretical resolution limit are discussed.

Fig. $6 \mathrm{~b}$ shows that for $x=0.10$ the two $\mathrm{Ru}-\mathrm{O}(1)$ first subshells appear to be different from each other above $T^{*} \approx 100$ $\mathrm{K}$, while they are not distinguishable at lower temperatures, indicating the appearance of a local distortion similar to that observed in the undoped material around $150 \mathrm{~K}$ (panel a, data from ref. 6). Anyway, at variance with the undoped case, no particular discontinuity can be seen in the $\mathrm{Ru}-\mathrm{Y}$ and $\mathrm{Ru}-\mathrm{Ru}$ bond lengths at the same temperature. Furthermore, Fig. $6 \mathrm{c}$ and $\mathrm{d}$ show that the same analysis for $x=0.15$ and 0.20 gives no evidence of such a structural transition, even in the first shell. In Fig. 7, $T^{*}$ is displayed as a function of $\mathrm{Zn}$ doping, showing that the order-disorder transition is not detected for $x>0.10$. This suggests that the $\mathrm{Zn}$ doping results in a local lattice distortion at any temperature. This finding could be explained by considering that the substitution on the A site introduces an additional short-range disorder which stabilizes the local octahedron distortion in the $\mathrm{Ru}-\mathrm{Ru}$ and $\mathrm{Ru}-\mathrm{Y}$ distances giving no temperature dependence. In addition, the $T_{\mathrm{DW}}$ peak is weakened for $x=0.10$ with respect to the undoped compound (Fig. 4) and gets completely lost for higher Zn doping together with $T^{*}$. Noteworthy, as we have shown above, ZF- $\mu$ SR measurements clearly indicate that the AFM transition is at play even for the highest $x=0.20$ sample. Indeed, the presence of $\mathrm{Zn}$ slightly reduces the $T_{\mathrm{N}, \mathrm{onset}}$ and broadens the magnetic transition. These results further emphasize the role of the local disorder induced by the $\mathrm{Zn}$ substitution, which could hide both

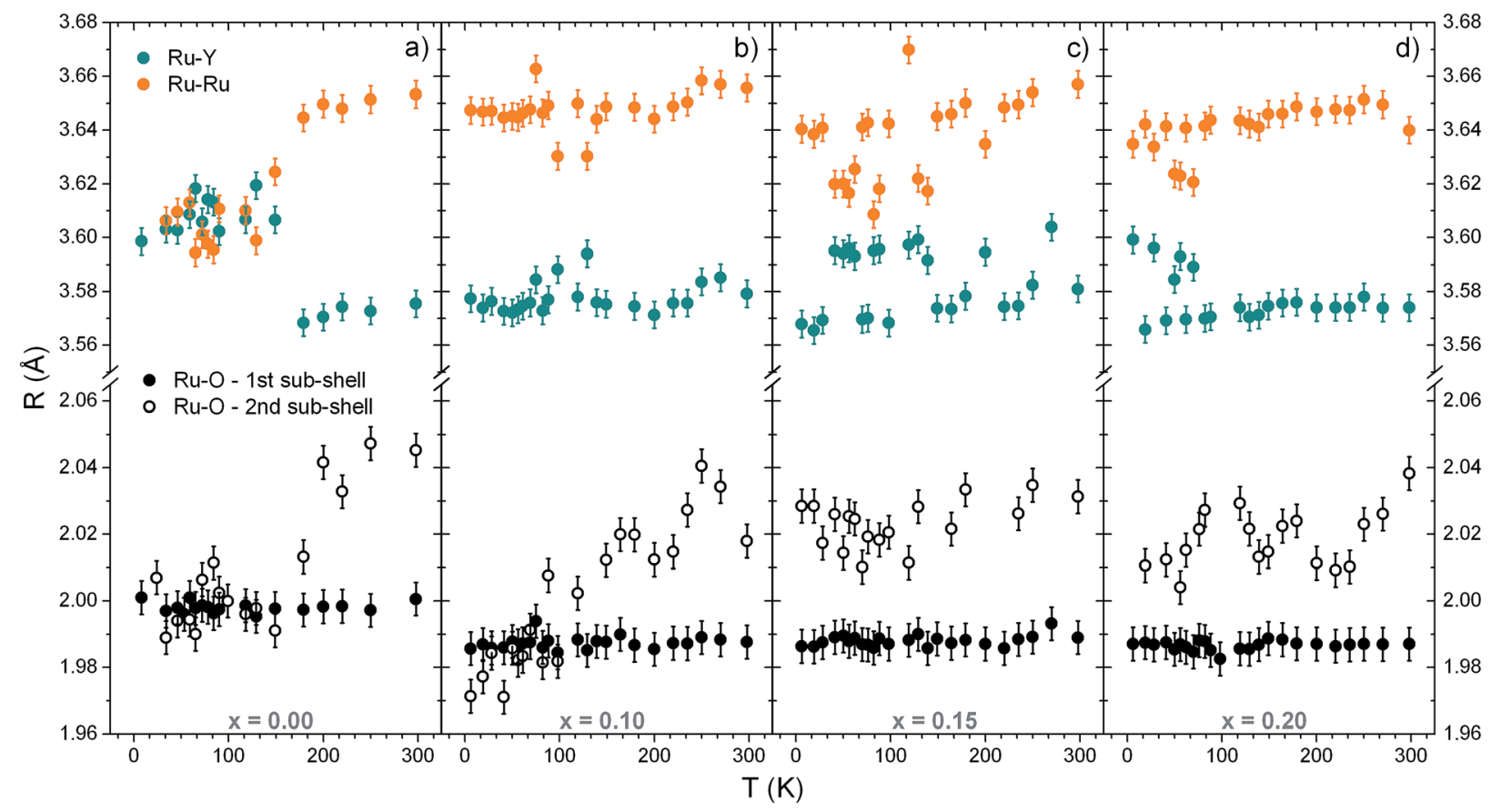

Fig. 6 Bimodal distribution of the Ru-O(1) bond lengths $\left(N_{\mathrm{Ru}-\mathrm{O}}=4\right.$ and $N_{\mathrm{Ru}-\mathrm{O}}=2$ for the shorter basal [solid black circles] and longer apical [open black circles] interatomic distances respectively) and second shell Ru-Y and Ru-Ru bond lengths, as a function of temperature for doping levels: (a) $x=0.00$ (data taken from ref. 6), (b) $x=0.10$, (c) $x=0.15$, and (d) $x=0.20$. 


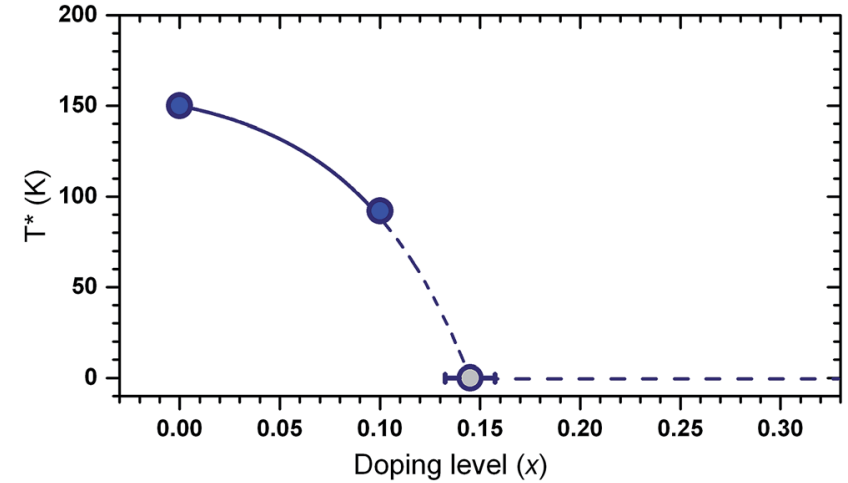

Fig. $7 T^{*}$ vs. $x$ experimental (blue circles) and estimated behavior (gray circle) for doping levels higher than 0.10. Data point size gives the experimental uncertainty on temperature. The continuous line is a guide to the eye.

the magnetoelastic effect at $T_{\mathrm{DW}} \approx T_{\mathrm{N}}$ and the order-disorder transition at $T^{*} \sim 2 T_{\mathrm{N}}$ for high $\mathrm{Zn}$ doping. The structural disorder induced by the heterovalent cationic substitution in the A site and observed by EXAFS on a short-range scale might have a twofold origin: a steric one, due to the difference of the $\mathrm{Zn} / \mathrm{Y}$ ionic size, and an electrostatic one, due to their different valence.

\section{Conclusions}

In conclusion, we gave evidence of the existence of a correlation between magnetic and structural transition temperatures in $\mathrm{Zn}$-doped $\mathrm{Y}_{2} \mathrm{Ru}_{2} \mathrm{O}_{7}$ pyrochlores, as far as the doping level is set below $x=0.15$. Higher dopings result in a local lattice distortion at any temperature, while the AFM transition is little affected by the amount of $\mathrm{Zn}$ substitution. The $\mathrm{Zn}^{2+}$ doping is thus believed to weaken the magnetoelastic coupling and to enforce and stabilize the distortion of the $\mathrm{RuO}(1)_{6}$ octahedra until the disappearance of the local order-disorder changeover.

\section{Acknowledgements}

We gratefully acknowledge the Spanish CRG at the ESRF for providing beamtime under experiment MA-2162. We are grateful to A. Amato and H. Luetkens of the Swiss Muon Source group at the PSI for technical support and to P. Carretta for fruitful discussions. CC received funding from Piano di Sviluppo di Ateneo UniMi 2014 - Linea B1.

\section{References}

1 J. S. Gardner, M. J. P. Gingras and J. E. Greedan, Rev. Mod. Phys., 2010, 82, 53-107.

2 R. C. Ewing, W. J. Weber and J. Lian, J. Appl. Phys., 2004, 95, 5949-5971.

3 M. T. Weller, R. W. Hughes, J. Rooke, C. S. Knee and J. Reading, Dalton Trans., 2004, 3032-3041.

4 L. Li and B. Kennedy, Chem. Mater., 2003, 15, 4060-4067.
5 R. Mani, M. Fischer, J. E. Joy, J. Gopalakrishnan and M. Jensen, Solid State Sci., 2009, 11, 189-194.

6 C. Castellano, G. Berti, S. Sanna, R. Ruiz-Bustos, J. van Duijn, A. Brambilla, A. Muñoz-Noval, P. Carretta, L. Duò and F. Demartin, Phys. Rev. B: Condens. Matter Mater. Phys., 2015, 91, 224101.

7 S. Yoshii and M. Sato, J. Phys. Soc. Jpn., 1999, 68, 3034-3040. 8 K. S. Lee, D. K. Seo and M. H. Whangbo, J. Solid State Chem., 1997, 131, 405-408.

9 M. K. Haas, R. J. Cava, M. Avdeev and J. D. Jorgensen, Phys. Rev. B: Condens. Matter Mater. Phys., 2002, 66, 094429.

10 S. Lee, J. G. Park, D. T. Adroja, D. Khomskii, S. Streltsov, K. A. McEwen, H. Sakai, K. Yoshimura, V. I. Anisimov, D. Mori, R. Kanno and R. Ibberson, Nat. Mater., 2006, 5, 471-476.

11 A. Yamamoto, P. A. Sharma, Y. Okamoto, A. Nakao, H. A. Katori, S. Niitaka, D. Hashizume and H. Takagi, $J$. Phys. Soc. Jpn., 2007, 76, 043703.

12 J. B. Goodenough, R. Manoharan and P. Paranthaman, J. Am. Chem. Soc., 1990, 112, 2076-2082.

13 J. Lee, S. Moon, T. Noh, T. Takeda, R. Kanno, S. Yoshii and M. Sato, Phys. Rev. B: Condens. Matter Mater. Phys., 2005, 72, 035124.

14 G. Berti, S. Sanna, C. Castellano, J. V. Duijn, R. Ruiz-Bustos, L. Bordonali, G. Bussetti, A. Calloni, L. Duò and A. Brambilla, unpublished work.

15 J. K. Gill, O. P. Pandey and K. Singh, Int. J. Hydrogen Energy, 2012, 37, 3857-3864.

16 B. Ismunandar, L. Li, B. J. Kennedy, K. Oikawa and T. Kamiyama, Phys. B, 2006, 385-386, 584-586.

17 S.-W. Chen, S.-W. Fu, C.-W. Pao, J.-M. Lee, S.-A. Chen, S.-C. Haw, J.-F. Lee, C.-H. Liu, C.-K. Chang, Y.-C. Chuang, H.-S. Sheu, K.-T. Lu, S.-T. Ku, L.-J. Chang and J.-M. Chen, Phys. Chem. Chem. Phys., 2015, 17, 23667-23673.

18 B. P. Mandal, A. Banerji, V. Sathe, S. K. Deb and A. K. Tyagi, J. Solid State Chem., 2007, 180, 2643-2648.

19 R. Kanno, Y. Takeda, T. Yamamoto, Y. Kawamoto and O. Yamamoto, J. Solid State Chem., 1993, 102, 106-114.

20 M. D. Field, B. J. Kennedy, B. A. Hunter and T. Vogt, J. Solid State Chem., 1999, 144, 467-469.

21 M. Kato, S. Harada, M. Fukatsu and K. Hirota, J. Phys.: Conf. Ser., 2011, 273, 012152.

22 M. Yasukawa, S. Kuniyoshi and T. Kono, Solid State Commun., 2003, 126, 213-216.

23 B. J. Kennedy, Acta Crystallogr., Sect. C: Cryst. Struct. Commun., 1995, 51, 790-792.

24 M. Ito, Y. Yasui, M. Kanada, H. Harashina, S. Yoshii, K. Murata, M. Sato, H. Okumura and K. Kakurai, J. Phys. Soc. Jpn., 2000, 69, 888-894.

25 B. Ravel and M. Newville, J. Synchrotron Radiat., 2005, 12, 537-541.

26 A. L. Ankudinov, B. Ravel, J. J. Rehr and S. D. Conradson, Phys. Rev. B: Condens. Matter Mater. Phys., 1998, 58, 75657576.

27 S. J. Blundell, T. Lancaster, P. J. Baker, W. Hayes, F. L. Pratt, T. Atake, D. S. Rana and S. K. Malik, Phys. Rev. B: Condens. Matter Mater. Phys., 2008, 77, 094424. 
28 C. Castellano, M. Ferretti, A. Martinelli and M. R. Cimberle, J. Alloys Compd., 2009, 478, 479-483.

29 A. Paolone, C. Castellano, O. Palumbo, F. Cordero, R. Cantelli, A. Vecchione, M. Gombos, S. Pace and M. Ferretti, Phys. C, 2007, 467, 167-173.

30 G. Beni and P. M. Platzmann, Phys. Rev. B: Solid State, 1976, 14, 1514-1518.

31 N. W. Ashcroft and N. D. Mermin, Solid State Physics, Saunders College, Philadelphia, 1976.
32 M. B. Johnson, D. D. James, A. Bourque, H. A. Dabkowska, B. D. Gaulin and M. A. White, J. Solid State Chem., 2009, 182, 725-729.

33 P. A. Lee, P. H. Citrin, P. Eisenberger and B. M. Kincaid, Rev. Mod. Phys., 1981, 53, 769-806.

34 T. Keiber, F. Bridges and B. C. Sales, Phys. Rev. Lett., 2013, 111, 095504. 